\title{
Spinocerebellar ataxia type 7 associated with pigmentary retinal dystrophy
}

\author{
A Michalik ${ }^{1}, \mathrm{~J}-\mathrm{J}$ Martin $^{2}$ and C Van Broeckhoven*,1
}

${ }^{1}$ Department of Molecular Genetics, Neurogenetics Group, Flanders Interuniversity Institute for Biotechnology (VIB), Antwerpen, Belgium; ${ }^{2}$ Laboratory of Neuropathology, Born-Bunge Foundation (BBS), University of Antwerp (UIA), Antwerpen, Belgium

Spinocerebellar ataxia type 7 (SCA7) is an autosomal-dominant, late-onset, slowly progressive disorder, primarily characterized by gradual loss of motor coordination, resulting from dysfunction and degeneration of the cerebellum and its connecting pathways. The disease is caused by expansion of a CAG trinucleotide repeat within the SCA7 gene, which encodes a polyglutamine tract within a novel protein, termed ataxin-7. The expansion of polyglutamine-encoding CAG repeats in dissimilar genes underlies eight neurodegenerative conditions besides SCA7, including a number of dominant ataxias related to SCA7. Although elongated polyglutamine itself can initiate neuronal dysfunction and death, its toxicity is modulated by the context of the disease proteins, as evidenced by the differing clinical and pathological presentation of the various disorders. In this respect, it is exciting that SCA7 constitutes the only polyglutamine disorder, in which the photoreceptors of the retina are also severely affected, leading to retinal degeneration and blindness. Since the discovery of the SCA7 mutation, numerous studies attempted to pinpoint the molecular mechanisms underlying the unique features of SCA7, particularly the retinal involvement. Here we summarize the clinical, pathological, and genetic aspects of SCA7, and review the current understanding of the pathogenesis of this disorder.

European Journal of Human Genetics (2004) 12, 2-15. doi:10.1038/sj.ejhg.5201108

Published online 22 October 2003

Keywords: spinocerebellar ataxia; spinocerebellar ataxia type 7; SCA7; ADCA type II; polyglutamine; neurodegeneration

\section{Introduction}

Autosomal-dominant cerebellar ataxias (ADCA) constitute a heterogeneous group of neurodegenerative disorders, clinically characterized by progressive incoordination of gait and limb movements (cerebellar ataxia), associated with variable additional symptoms, including dysarthria, dysphagia, opthalmoplegia, pyramidal and extrapyramidal signs, dementia, or retinopathy. Pathologically, ADCA are

*Correspondence: Prof Dr C Van Broeckhoven, Department of Molecular Genetics VIB8, University of Antwerp (UIA), Universiteitsplein 1, B-2610 Antwerpen, Belgium. Tel: +32382026 01; Fax: + 32382025 41; E-mail: christine.vanbroeckhoven@ua.ac.be

Received 17 June 2003; revised 31 August 2003; accepted 11 September 2003 typified by prominent atrophy of the cerebellum, brainstem and the spinal cord, together with differing degree of degeneration in the cerebral cortex and basal ganglia. The onset is usually in the third to fifth decade, but genetic anticipation - a tendency for earlier onset and increased phenotypic severity in younger generations - is frequently noted in ADCA families. A meaningful classification of ADCA was hampered by the extreme inter- and intrafamilial variability of associated clinical features, and was ultimately provided only by genetic linkage studies. Nevertheless, one subtype of ADCA - first described in $1937^{1}$ and designated ADCA type $\mathrm{II}^{2}$ or OPCA type $\mathrm{III}^{3}$ - was clearly distinguished from all others by the invariable presence of pigmentary retinal dystrophy. This subtype later became known as SCA7 (for spinocerebellar ataxia type 7), after 
genetic studies defined its locus on chromosome 3 . In this review, we will outline the clinical, pathological, and genetic aspects of SCA7, and then focus on recent advances in understanding the pathogenesis of this disorder.

\section{Clinical and pathological features of SCA7}

The first neurological sign of SCA7 is typically gait ataxia. Limb ataxia develops over the following years and is invariably associated with dysarthria. Additional symptoms frequently comprise slowing of saccades, opthalmoplegia, dysphagia, and pyramidal signs. In contrast, extrapyramidal signs are less common, and dementia and hearing loss are rare. ${ }^{4-14}$ Pathologically, SCA7 presents as olivocerebellar atrophy with additional degenerative changes in the upper cervical spinal cord and pallidal and subthalamic systems (Figure $1 \mathrm{a}-\mathrm{c}){ }^{4,5,7}$ The cerebellum shows an extensive loss of Purkinje cells (Figure 1g, h), neuronal loss and gliosis in dentate nuclei, and milder changes in granule cell layer. Moreover, there is a dramatic neuronal loss and gliosis in the inferior olivary nuclei of medulla oblongata, variable but often mild loss of neurons in basis pontis, and atrophy of the spinocerebellar tracts.

Nevertheless, the most conspicuous feature of SCA7 is progressive visual impairment. Central vision is compromised first, but the condition evolves toward complete blindness. ${ }^{2,4,5}$ Importantly, asymptomatic visual defects such as impairment of color vision in the blue-yellow axis - might arise years before the visual failure is noticed. Degenerative changes in the retina initially affect cone photoreceptors, but progress toward a cone-rod dystrophy. ${ }^{4,5,7}$ Fundoscopy reveals an early loss of normal foveal reflex, followed by granular pigmentation of the macula interspersed with pale areas of pigmentary atrophy (Figure 1d-f). Histology shows complete absence of photoreceptors, severe loss of ganglion cell neurons, and thinning of both nuclear and plexiform layers (Figure 1i, j). Moreover, there is migration of melanin pigment from the retinal epithelium toward the atrophic retina.

\section{Onset age}

The onset of SCA7 is usually in the second to fourth decade (Median, 30 years; IQR, 18-41 years), but can vary from a few months to well over 70 years (Figure 2). Importantly, SCA7 displays marked anticipation of both onset age and rate of progression: first, the symptoms appear on average 20 years earlier in the offspring of affected parents than in the parents themselves ${ }^{10,11}$; second, progression to death is faster in patients with onset below 10 years $(6 \pm 6$ years) than in patients with later onset $(20 \pm 10$ years $) .{ }^{15}$ Intriguingly, cases with infantile onset - below 2 years of age occasionally arise in the progeny of SCA7 fathers. $4,12,16,17$ These cases manifest with failure to thrive, weight loss, weakness, and hypotonia, and rapid progression to death. Moreover, these patients often show patent ductus arteriosus, suggesting involvement of heart tissue.

\section{Genetics of SCA7}

The characteristic presence of retinopathy indicated that SCA7 was genetically distinct from other spinocerebellar ataxias. Indeed, SCA7 families did not show linkage to other known loci for spinocerebellar ataxia, ${ }^{5-7}$ and a novel SCA7 locus was later identified on chromosome 3p12-

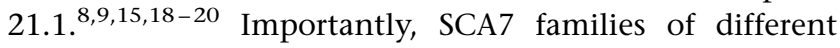
geographic origin all showed linkage to this locus, indicating that the disease was genetically homogeneous (hitherto, only one family with clinical assessment of SCA7 was reported not to segregate the $S C A 7$ mutation $^{13}$ ).

By that time it had become obvious that a number of progressive neurodegenerative conditions with anticipation - including other spinocerebellar ataxias - were caused by expansion of unstable CAG trinucleotide repeats coding for polyglutamine within the respective proteins. ${ }^{21}$ This strongly suggested that CAG expansion might also underlie SCA7. Indeed, an unknown expanded CAG repeat cosegregated with the disease in some SCA7 families, ${ }^{22}$ and a novel polyglutamine-containing protein was detected in lymphoblasts from several SCA7 patients ${ }^{23,24}$. Therefore, a search for expanded CAG repeats from within the SCA7 candidate region was initiated, and eventually led to identification of the SCA7 gene. ${ }^{25-27}$ The gene encodes a novel protein of 892 amino acids, named ataxin7, carrying an N-terminal polyglutamine tract specified by the SCA7 CAG repeat. The gene consists of 13 exons and spans $140 \mathrm{~kb}$ of genomic DNA. ${ }^{28}$

The identification of the SCA7 gene enabled estimating the frequency of SCA7 relative to other forms of ADCA. In general, ADCA are fairly rare disorders with prevalence of 0.3-2 per 100000 . Approximately, two-thirds of all ADCA cases are explained by a CAG expansion in the known spinocerebellar ataxia genes (SCA1, SCA2, SCA3/MJD-1, SCA6, and SCA7). ${ }^{29}$ SCA7 is a rare form of ADCA, accounting for $1-11.7 \%$ of genetically diagnosed ADCA in diverse populations. ${ }^{17,30-38}$ However, the relative frequency of SCA7 is considerably higher in certain regions due to local founder effects - for example, in Scandinavia, it constitutes the most common form of ADCA. ${ }^{39}$

\section{Genotype-phenotype correlations}

The expansion of a coding CAG repeat causes eight progressive neurodegenerative conditions in addition to SCA7, including Huntington's disease (HD), spinal and bulbar muscular atrophy (SBMA), dentatorubral and pallidoluysian atrophy and other spinocerebellar ataxias - SCA1, SCA2, SCA3/MJD, SCA6, and SCA17. ${ }^{40}$ In all these disorders, the wild-type alleles typically contain less than $36 \mathrm{CAG}$, whereas mutant alleles carry from 36 up to several hundred CAG (with exception of $\mathrm{SCA}^{41}$ ). Anticipation in these diseases is explained by the increased tendency of an expanded CAG repeat to undergo further meiotic expansion, together with the ability of longer 

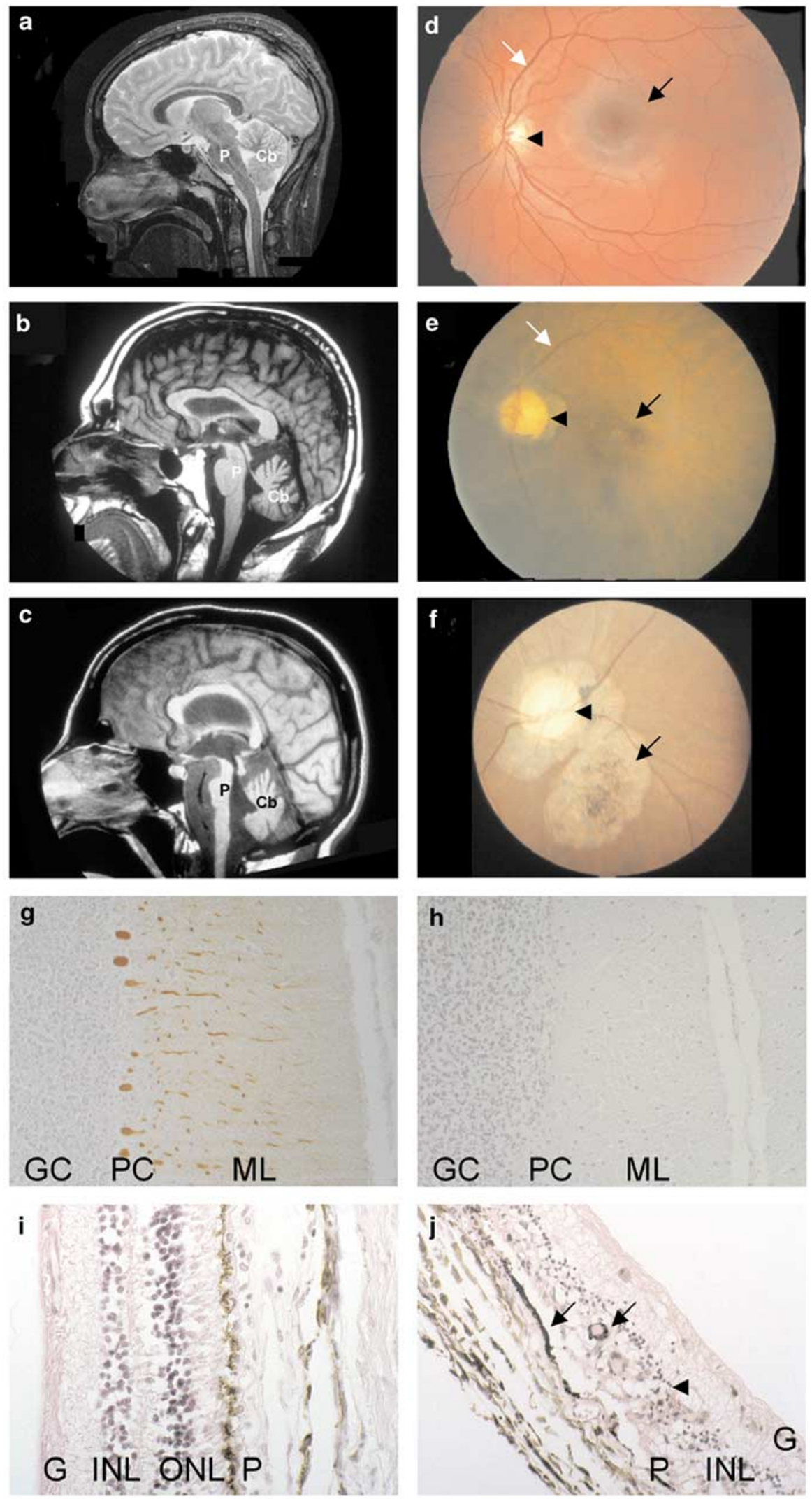


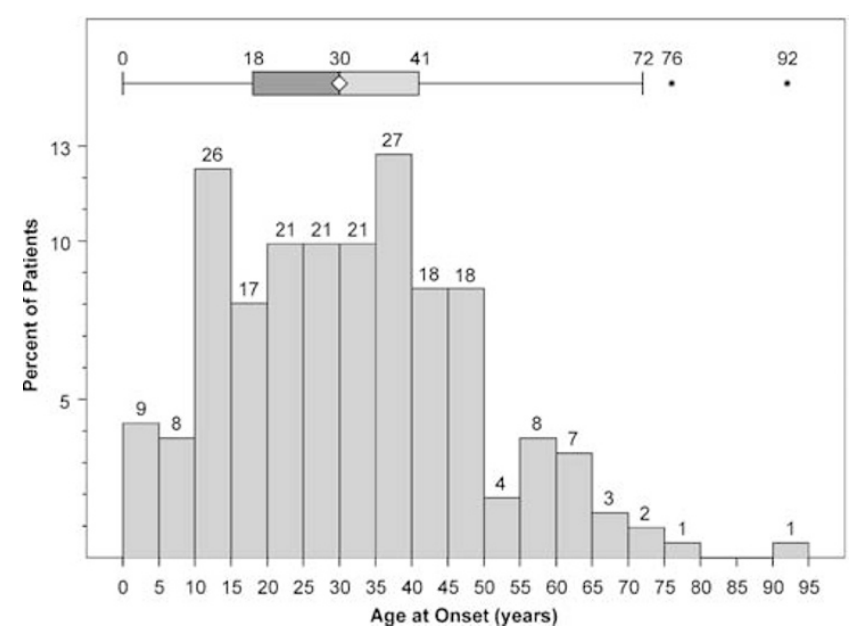

Figure 2 SCA7 age at onset. Histogram representation of onset ages of 212 SCA7 patients described in references 10-14,16,17. Digits above the bars specify the actual number of patients in each age category. Above the histogram, the data is summarized in a box-whisker plot, indicating the Median (diamond) and the IQR (filled bar); the whiskers are drawn up to the farthest value within $1.5 \times$ IQR distance from the quartiles; values outside of this range (outliers) are marked with asterisk.

repeats to provoke earlier onset and augmented disease severity.

The SCA7 CAG repeat is polymorphic and mostly contains 4-18 CAG in controls, with $70-80 \%$ of alleles carrying 10 CAG. A tiny fraction of the wild-type alleles $(<0.5 \%)$ contain $28-35$ CAG. $^{42}$ These 'intermediate' alleles do not cause disease (although mild symptoms were reported in some patients with $34-36 \mathrm{CAG}^{27,43}$ ), but show

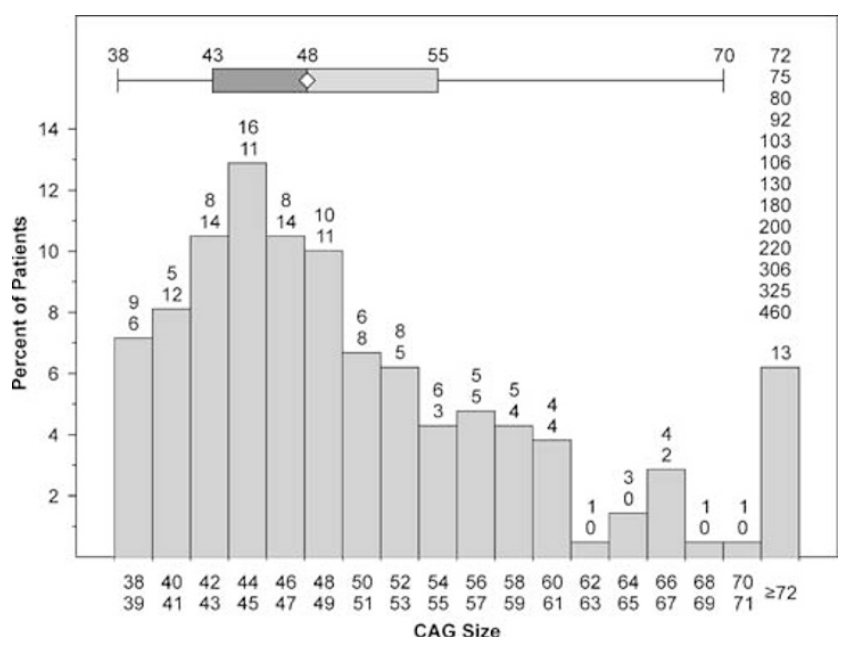

Figure 3 SCA7 CAG repeat size. Histogram representation of CAG repeat size in 212 SCA7 patients described in references 10-14,16,17. Digits above the bars specify the actual number of patients carrying each repeat length. Above the histogram, the data is summarized in a boxwhisker plot, indicating the Median (diamond) and the IQR (filled bar); the whiskers are drawn up to the farthest value within $1.5 \times$ IQR distance from the quartiles; values outside of this range ( $\geqslant 72 \mathrm{CAG}$ ) are listed individually.

enhanced probability of expanding into the disease range upon paternal transmission. Thus, they constitute a pool of mutation-prone alleles on which de novo expansions may arise, leading to re-emergence of the disease. Mutant SCA7 alleles typically contain between 38 and 70 repeats (Median, 48 CAG; IQR, 43-55 CAG), but extreme expansions - up to 460 CAG - have been documented (Figure 3). ${ }^{12,16,17}$

Figure 1 Pathological hallmarks of SCA7. (a-c) Magnetic resonance imaging of a control individual (a) and two SCA7 patients $(\mathbf{b}, \mathbf{c})$. The patient in (b) displays severe atrophy of the cerebellum $(\mathrm{Cb})$, but almost complete sparing of the pons $(\mathrm{P})$. This patient carried 38 CAG repeats in the SCA7 gene, and, at the time of examination (60 years), exhibited mild unsteadiness of gait. In contrast, the patient in (c) shows very severe cerebellar atrophy, as well as marked atrophy of the pons (P). This individual harbored 52 CAG repeats and was examined at 40 years of age. (d-f) Fundoscopy images of a control individual (d) and two SCA7 patients $(\mathbf{e}, \mathbf{f})$. Note the normal appearance of the macula (black arrow) and the optic disk (arrowhead), and the well-developed vasculature (white arrow) in the control individual (d). On the contrary, the retina of patient (e) displays an atrophic macula (black arrow), very pale optic disk (arrowhead), and poor vasculature (white arrow). This patient carried 52 CAG repeats and was examined at 40 years. Moreover, the retina of patient (f) shows an extremely pale optic disc (arrowhead), and atrophy of the pigmentary epithelium and choroid layer (arrow). This patient was diagnosed with gait ataxia, dysarthria, and decreased eyesight at the age of 20 years, and died at 28 years. The number of CAG repeats in this individual is unknown. (g, h) Histology of the cerebellar cortex of a control individual (g) and an SCA7 patient (h), stained with an antibody against calbindin to visualize Purkinje neurons. Note the intensely labeled bodies of Purkinje cells (PC), and their dendritic extensions within the molecular layer (ML) in the control cerebellum $(\mathbf{g})$. In contrast, the cerebellum of a SCA7 patient (h) displays a dramatic loss of Purkinje neurons. The patient carried 55 CAG repeats, developed ataxia and eyesight defects at 20 years, and died at 45 years. (i, j) Histology of the retina of a control individual (i) and an SCA7 patient (j). The control retina (i) shows a proper organization of the retinal layers: the pigment epithelium $(P)$ in the middle of the picture, the nuclei of rods and cones within the outer nuclear layer (ONL), the nuclei of bipolar neurons within the inner nuclear layer $(\mathrm{INL})$, and the ganglion cell layer (G). On the contrary, the retina of the SCA7 patient (j) is severely degenerated, and displays complete demise of photoreceptor segments and nuclei, disorganization of the INL (arrowhead), and migration of the melanin pigment deep into the atrophic retina (arrows). The patient was as in (f). 


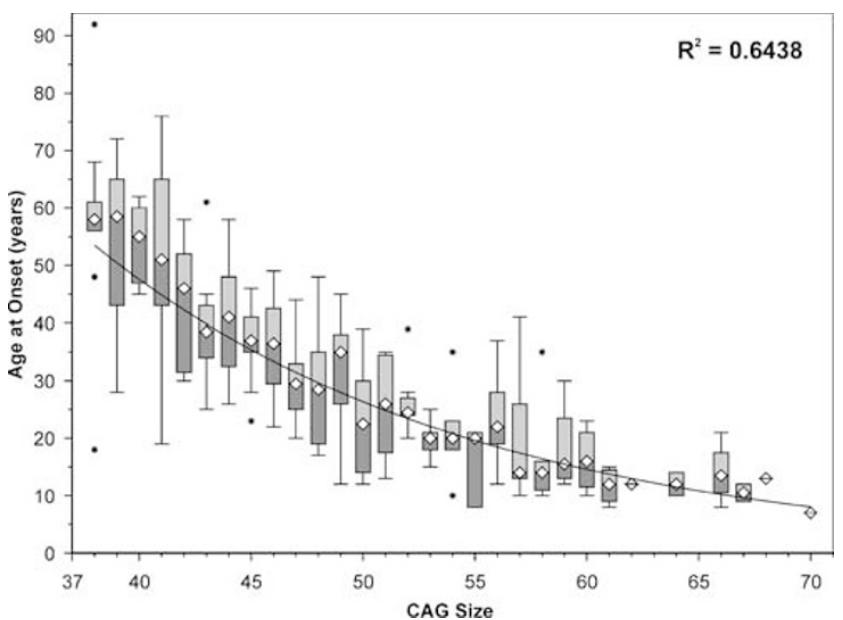

Figure 4 CAG repeat size - age at onset correlation in SCA7. The chart depicts the correlation between the SCA7 CAG repeat size and onset age in 198 patients, carrying between 38 and 70 CAG, reported in references 10-14,16,17. For each repeat size, the observed onset ages are summarized in a box-whisker plot, indicating the Median (diamond) and the IQR (filled bar); the whiskers are drawn up to the farthest value within $1.5 \times \mathrm{IQR}$ distance from the quartiles; values outside of this range (outliers) are marked with asterisk. An exponential best-fitting curve and its corresponding $R^{2}$ value are also indicated.
The length of the SCA7 CAG repeat is inversely correlated with onset age: in the range between 38 and $70 \mathrm{CAG}$, the repeat size accounts for $64 \%$ of the observed variance in the age at onset (Figure 4). Repeats larger than 70 CAG typically result in onset below 10 years of age, while repeats longer than 130 CAG lead to onset age of less than 2 years. The repeat length is also negatively correlated with disease duration until death: while the disease progresses over several decades in patients with relatively short CAG expansions, it displays dramatically accelerated course in juvenile ( $>70$ CAG) and infantile (>200 CAG) cases, resulting in death within a few years or months, respectively. Finally, the CAG length also dictates the initial symptom: patients presenting with cerebellar ataxia tend to carry shorter repeats than those presenting with macular degeneration. ${ }^{10,12,13}$ To sum up, longer CAG tracts lead to earlier onset, more rapid progression, and increased chance of visual failure preceding cerebellar ataxia.

\section{Dynamics of the SCA7 CAG repeat}

The striking degree of anticipation in SCA7 must reflect profound meiotic instability of the SCA7 CAG repeat. We examined transmission of the $S C A 7$ CAG repeat in a multigenerational pedigree previously identified by our laboratory $\left(\mathrm{CA}^{5}\right)$, and in one smaller SCA7 family (CA6) (Figure 5). We then combined our data with existing

\section{Family CA1}

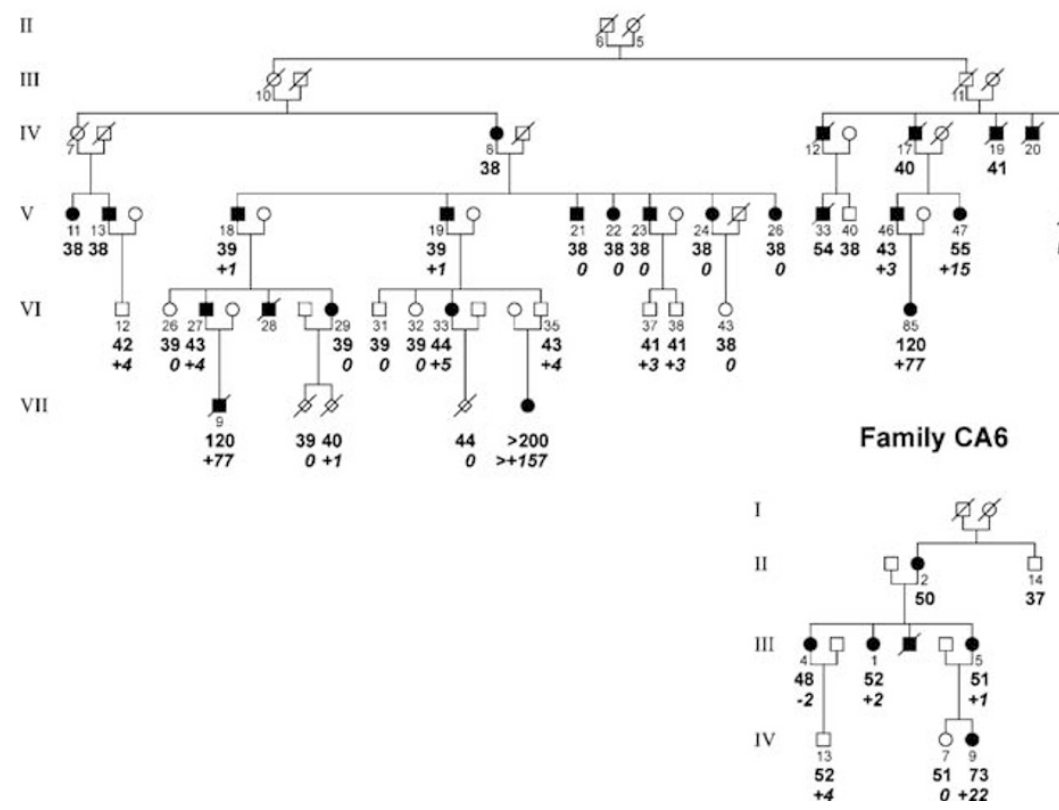

Figure 5 Belgian SCA7 families CA1 and CA6. A simplified representation of the Belgian SCA7 families CA1 and CA6, depicting only the carriers of the expanded CAG repeat. The size of the expanded CAG repeat in each individual is shown in bold, while the size change upon each transmission is indicated in bold italics. Detailed clinical and neuropathological assessment of the CA1 family members has been previously reported. ${ }^{5,14}$ The numbering of individuals in this pedigree follows the one in reference 5 . 
information on the transmission behavior of the SCA7 CAG repeat. ${ }^{10-13,16,17}$ Our analysis demonstrated that the expanded SCA7 CAG repeat is altered at almost every transmission (87.5\% paternal and $84.5 \%$ maternal), and that most such alterations are further expansions $(83.3 \%$ in paternal and $73.8 \%$ in maternal transmission) (Figure 6).

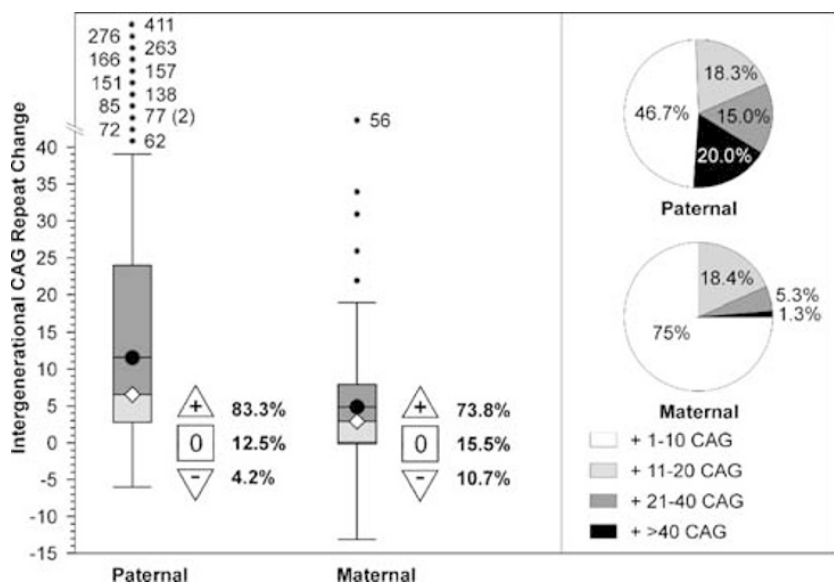

Figure 6 Intergenerational CAG repeat change. Left: Boxwhisker plot representation of intergenerational CAG repeat changes in 55 paternal and 89 maternal transmissions in the Belgian families CA1 and CA6, and in families described in references $10-13,16,17$. The data indicate the Median intergenerational change (diamond) and the corresponding IQR (filled bar); the whiskers are drawn up to the farthest value within $1.5 \times I Q R$ distance from the quartiles; values outside of this range (outliers) are marked with asterisk; in addition, a filled circle indicates the Median expansion size. To the right of each box plot, the proportions of expansion, contraction, and 'no change' are shown. Right: Pie diagram representation of the size distribution of expansions in paternal and maternal transmissions.
Moreover, expansions arising on paternal alleles are typically larger than those occurring on maternal alleles (Median, + 11.5 versus + 5 CAG) (Figure 6), despite the fact that in the analyzed sample the paternal alleles were on average smaller than the maternal ones $(42.5 \pm 3.72$ versus $44.9 \pm 4.40 \mathrm{CAG}$, respectively) (Table 1 ). In addition, we noticed that alleles, which do undergo a size change upon transmission, are mostly larger than the ones remaining unaltered, although this difference was statistically significant only for maternal alleles (Table 1). In contrast, there exists no difference in size of parental alleles undergoing expansion and those undergoing contraction (Table 1). Finally, in both genders, we observed a statistically significant tendency for larger expansions $(>+20$ CAG) to arise on longer parental alleles (Table 1). Interestingly, however, paternal alleles that give rise to expansions of more than $+40 \mathrm{CAG}$ are not significantly larger than those on which expansions of between +21 to +40 CAG occur. Hence, once a paternal SCA7 CAG repeat reaches a certain threshold $(44.7 \pm 3.13 \mathrm{CAG})$, it acquires the ability of extreme meiotic expansions ( $>+40$ CAG; see below).

Importantly, the marked tendency toward larger expansions in paternal than in maternal transmission would predict more pronounced anticipation in paternal transmission. However, although earlier work observed similar effect $^{6,15}$, later studies using larger groups of patients did not detect any difference in anticipation between genders. ${ }^{10,11}$ Nevertheless, the largest expansions - causing juvenile or infantile onset - occur exclusively in paternal transmission. ${ }^{12,16,17}$ Intriguingly, these massive expansions (up to $>+400$ CAG) arise on moderately sized paternal alleles (40-55 CAG), further underscoring the exceptional instability of the $S C A 7$ CAG repeat in male meiosis. Indeed, such instability was confirmed by typing individual sperm of SCA7 males carrying 46 or 53 CAG. $^{44}$ In $99 \%$ of sperm,

Table 1 Dependence of intergenerational change of the SCA7 CAG repeat on parental allele size

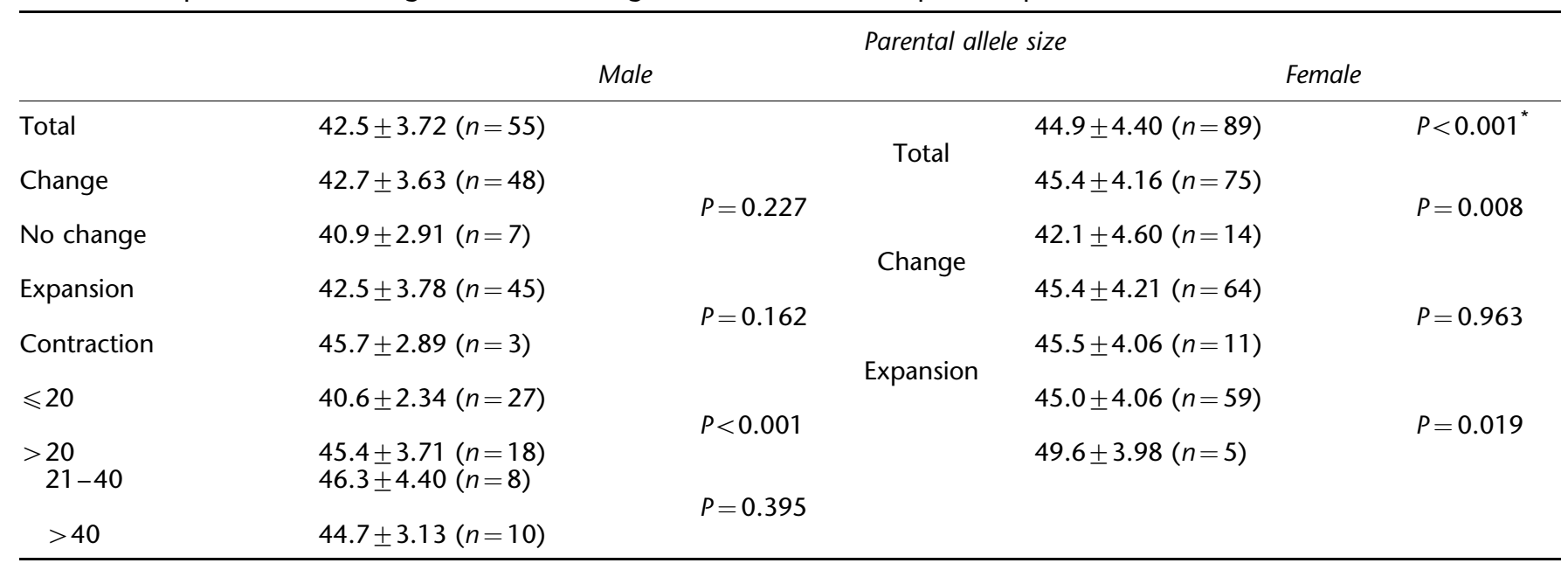

* $P$-values were obtained using a Student's $t$-test. 
the repeat further expanded and on average gained additional +104 or +136 CAG, respectively. Yet, such huge expansions are only sporadically noted in the progeny of carrier fathers $(<15 \%$ have repeats longer than 100 CAG). Therefore, these extremely long repeats likely compromise fertilization, or lead to embryonic lethality, ${ }^{44}$ an interpretation also supported by the observed bias toward females for being the actual transmitting parent in SCA7. ${ }^{11}$

Compared to other CAG repeats, the SCA7 CAG repeat shows the greatest 'expandability'. ${ }^{45}$ This measure reflecting both the likelihood and the magnitude of further meiotic expansion - is largely determined by the genomic context of a particular CAG repeat. It is higher for repeats residing in regions with elevated GC content and/or within predicted CpG islands. Indeed, the SCA7 CAG repeat lies in a very GC-rich region $(83.5 \%$ over $100 \mathrm{bp})$ and within a putative $\mathrm{CpG}$ island. ${ }^{45}$ Importance of genomic context for meiotic instability of the SCA7 CAG repeat was recently demonstrated in transgenic mice. ${ }^{46}$ Mice carrying an SCA7 cDNA transgene showed low rate of intergenerational repeat changes, minor alterations, and a bias toward contractions. In contrast, animals bearing a $13.5-\mathrm{kb}$ genomic fragment encompassing the SCA7 CAG repeat displayed size alterations at most transmissions, larger changes, and a clear bias toward expansions. Moreover, this instability was significantly reduced by deletion of genomic sequences downstream of the CAG repeat, indicating that this region harbors some of the cis-elements triggering the instability.

The SCA7 CAG repeat also displays a considerable degree of somatic instability with a clear bias toward expansion. ${ }^{10,11,25,26,44}$ Increments of up to +10 CAG are frequent in blood, muscle, and buccal cells, while additions of $>+100$ CAG are noted occasionally. Interestingly, in transgenic mice carrying the genomic SCA7 CAG repeat, moderate expansions were detected in all tissues, but large increases (up to $+150 \mathrm{CAG}$ ) were confined to brain and liver. $^{46}$

\section{Ataxin-7}

Ataxin-7 is an 892-amino-acid protein with unknown function and no overall similarity to other proteins. ${ }^{25}$ The polyglutamine tract is located at its very $\mathrm{N}$-terminus between codons 30-39 and is preceded by an imperfect polyalanine repeat. Furthermore, a proline-rich region comprising several predicted SH3-binding domains is located downstream of the polyglutamine repeat, and participates in protein interactions. ${ }^{47}$ Ataxin-7 carries a functional nuclear localization signal (NLS) between amino acids 378 and 394, and might also include a nucleolar localization signal (NoLS) between amino acids 357 and $412{ }^{48}$ Finally, ataxin-7 contains a motif (amino acid 341384) with similarity to the phosphate-recognition site of arrestins, which might be involved in phosphorylation- dependent interaction of ataxin-7 with yet unknown proteins. ${ }^{49}$ Intriguingly, this interaction might regulate the subcellular localization of ataxin-7 since the putative phosphate-binding site overlaps with the NLS. Ataxin-7 shows remarkable conservation $(88.7 \%)$ between mice and humans. ${ }^{50}$ In particular, its NLS and the arrestin-homology domain are almost entirely conserved, suggesting that they are central to ataxin-7 function. On the contrary, no homologue of ataxin-7 is found in Drosophila or Caenorhabditis elegans.

\section{Pathogenesis of SCA7}

As pointed out already, expansion of polyglutamineencoding CAG repeats causes eight neurodegenerative conditions besides SCA7. Polyglutamine expansion represents a dominant toxic gain-of-function mutation, leading to neuronal dysfunction and degeneration regardless of the precise protein context within which it resides. ${ }^{51}$ The toxic effects of expanded polyglutamine might derive from its propensity to form aggregates, but the exact mechanisms of polyglutamine pathogenesis are largely unknown (for a thorough discussion of this issue, see references Zoghbi and Orr, ${ }^{40}$ Gusella and MacDonald, ${ }^{52}$ and Tobin and Signer ${ }^{53}$ ). Importantly, however, the different diseases are clinically distinct and display characteristic patterns of neuronal loss. ${ }^{54}$ Hence, the protein context must modulate polyglutamine toxicity and determine which neurons will degenerate in each disease. In the following, we inspect which attributes of ataxin-7 might underlie the unique features of SCA7 pathogenesis.

Expression levels The most evident manner as to how the toxicity of ataxin-7 could be restricted to select neuronal populations is if the mutant protein was expressed preferentially in these neurons. In contrast, SCA7 mRNA is present at roughly equal quantity in all neuronal populations. ${ }^{55}$ Moreover, the amount of ataxin-7 protein - while exhibiting considerable variation between distinct brain regions - is not markedly elevated in neurons vulnerable to SCA7. ${ }^{55-58}$

Subcellular localization Another way in which ataxin-7 protein context might modulate polyglutamine toxicity in SCA7 is by dictating the subcellular localization of the mutant protein - in particular, targeting of expanded polyglutamine to the nucleus may greatly aggravate its toxicity. ${ }^{59-61}$ Several observations proposed that ataxin-7 was primarily a nuclear protein: it carries a functional $\mathrm{NLS} ;{ }^{48}$ the mutant protein is enriched in the nuclei of patients' lymphoblasts; ${ }^{24}$ and both wild-type and mutant proteins localize into the nuclei of transfected cells. ${ }^{48,62}$ However, the subcellular localization of ataxin-7 in neurons proved more complex: it appears mainly cytoplasmic, sometimes nuclear, and often both. ${ }^{58-58}$ Moreover, incidence of these diverse immunoreactivity patterns 
varies both between and within distinct brain regions and exhibits age-related changes in some neuronal types - most remarkably cerebellar Purkinje cells ${ }^{58}$ and retinal photoreceptors. ${ }^{63}$ Yet, subcellular localization of ataxin-7 in SCA7-vulnerable neurons is not considerably different from its localization in spared cells.

Recently, Einum et $a l^{64}$ described a novel isoform of ataxin-7, which contains an alternative C-terminus, resulting from incorporation of an extra exon into the SCA7 mRNA. Intriguingly, this isoform is enriched within the CNS and primarily localizes into neuronal cytoplasm. Nevertheless, it is unclear to what extent the two ataxin7 variants differ with respect to SCA7 pathogenesis.

Aggregation Like other polyglutamine disease proteins, mutant ataxin-7 forms prominent aggregates within neuronal nuclei - nuclear inclusions (NI) (Figure 7). ${ }^{56-}$ $58,65,66$ Importantly, ataxin-7 NI are commonly found both in affected regions, including inferior olives and retinal photoreceptors, and in spared areas, such as the cerebral cortex. In contrast, NI are largely absent from cerebellar
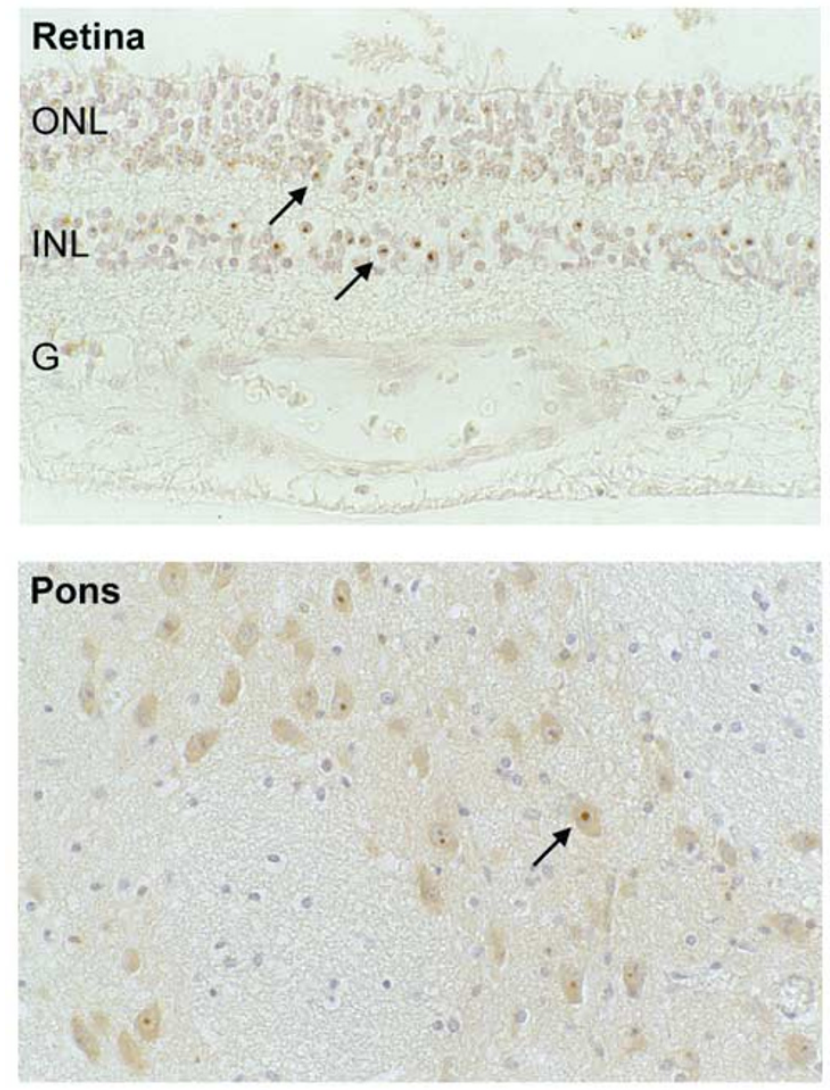

Figure 7 SCA7 NI. NI (arrow) of mutant ataxin-7, detected using the CM189 antibody ${ }^{66}$ in the retina and pons of a juvenile-onset SCA7 patient (individual CA1VI.85; 120 CAG; onset, 3 years). G, ganglion cell layer; $\mathrm{INL}$, inner nuclear layer; ONL, outer nuclear layer.
Purkinje cells, although these neurons are severely affected in SCA7. Finally, Einum et $a l^{57}$ reported a late-onset SCA7 case without any discernible NI. Together, this lack of correlation between neurons harboring ataxin-7 NI and neurons vulnerable to SCA7 suggests that NI are neither sufficient nor necessary to induce neuronal dysfunction and degeneration in SCA7.

Yet, it is too early to reject a causative role for aggregation in SCA7 pathogenesis. First, without detailed understanding of the mechanisms leading to polyglutamine toxicity, one should not assume that distinct neuronal types are equally vulnerable to polyglutamine aggregates. Second, because post-mortem analysis only offers an end-stage view of this slowly progressive condition, it remains possible that ataxin-7 aggregates appear earlier - and thus elicit toxicity for longer - in affected neurons. Third, neurons that do not develop aggregates might still degenerate due to transneuronal insults. Lastly, the presence of aggregates is typically evaluated using light microscopy, which reliably detects large NI, but might easily overlook more minute, although still very harmful aggregates. ${ }^{67}$ Therefore, the study of ataxin-7 aggregation will remain crucial for understanding the SCA7 pathogenesis.

Turnover Polyglutamine expansion might diminish the turnover of certain disease proteins: an often-quoted example is that of mutant ataxin-1, which is degraded three times less efficiently than its wild-type counterpart in vitro. ${ }^{68}$ Importantly, reduced turnover would increase the cellular load of expanded polyglutamine, thereby accelerating its aggregation and toxicity. In addition, disproportionate accumulation of disease proteins might disturb cellular functions in which they usually participate.

Yvert et $a l^{69}$ examined the metabolism of ataxin-7 in SCA7 transgenic mice (B7E) with widespread neuronal expression of the protein. Intriguingly, whereas the wildtype protein was readily degraded, the clearance of mutant ataxin-7 was markedly reduced. However, this disparity in turnover of wild-type versus mutant ataxin-7 was not replicated in transiently transfected COS cells, nor in photoreceptors of another transgenic model, both of which expressed much higher amounts of the protein. Hence, further experiments are needed to elucidate the mechanisms that stabilize mutant ataxin-7 in neurons. Nevertheless, Yvert et al ${ }^{69}$ did not report any significant differences in the turnover of mutant ataxin-7 between distinct neuronal populations of these transgenic mice.

Proteolytic processing SCA7 NI only contain N-terminal fragments of mutant ataxin-7, suggesting that proteolytic cleavage of ataxin-7 might facilitate its aggregation. ${ }^{56,66}$ Indeed, a short N-terminal fragment of mutant ataxin-7 aggregates more readily than the full-length protein in transfected cells. ${ }^{62}$ In addition, ataxin-7 inclusions arise exclusively in the nucleus, even in neurons with primarily 
cytoplasmic localization of the full-length protein. ${ }^{56}$ Hence, truncation of ataxin-7 might further promote its localization within the nucleus, where it perhaps exhibits faster aggregation and greater toxicity. Importantly, restricted occurrence of such proteolytic events might partly determine the selective patterns of neurodegeneration in SCA7.

To explore this latter possibility, Yvert et al ${ }^{69}$ inspected the proteolysis of ataxin-7 in the B7E SCA7 transgenic mice. As mentioned above, mutant ataxin-7 displays reduced turnover and increased accumulation in neurons of these animals. Intriguingly, however, the mutant protein does not remain full-length - rather, its N-terminal fragments progressively accumulate in neuronal nuclei and eventually form nuclear inclusions. Importantly, these NI develop with similar timing in most neurons of these mice, arguing that distinct neuronal types process mutant ataxin-7 identically.

Ataxin-7 interaction partners Perhaps the most appreciated way in which protein context might modulate polyglutamine toxicity is by mediating interactions with other cellular proteins. Such interactions would likely influence the subcellular localization, metabolism, and aggregation of mutant ataxin-7, and might in part specify which cellular proteins will be recruited into the resulting aggregates. Still more interestingly, polyglutamine expansion might affect the strength of ataxin-7's interactions with its usual binding partners, this way impinging on normal cellular functions of the protein. Therefore, identification of ataxin-7's binding partners will be essential for unraveling the various pathways involved in SCA7 pathogenesis.

To isolate novel interaction partners of ataxin-7, two groups carried out yeast two-hybrid screens. Lebre et al ${ }^{47}$ reported interaction of ataxin-7 with a group of nine SH3domain-containing proteins generated by alternative splicing of a single gene, SH3P12. Although all nine isoforms are capable of binding ataxin-7, it is unclear which interactions actually take place in vivo. The SH3P12 proteins engage in various cellular processes including actin stress fiber formation and cell-cell adhesion. However, the function of most isoforms is unknown and no role in CNS has been ascribed to any of them. Although the SH3P12 proteins as a group are expressed ubiquitously, select isoforms might perhaps be enriched in SCA7 vulnerable neurons. Importantly, the SH3P12 proteins are sequestered into SCA7 NI, encouraging further analysis of their link to SCA7 pathogenesis.

Matilla et $a l^{70}$ demonstrated that ataxin-7 could associate with the S4 subunit of the $19 \mathrm{~S}$ regulatory particle (RP) of the proteasome, and that this interaction became weaker with increasing polyglutamine length in ataxin-7. Intriguingly, in SCA7 brains the amount of S4 subunit was reduced selectively in affected neurons. The authors hypothesized that binding of ataxin-7 might either stabilize the S4 subunit or assist its incorporation into the 19S RP. Consequently, if this interaction were disrupted by polyglutamine expansion in ataxin-7, this would destabilize the S4 subunit, leading to dysfunction of the proteasome. In turn, this might promote the accumulation and aggregation of mutant ataxin-7, thus aggravating its toxicity. Nevertheless, this attractive loss-of-function hypothesis awaits further scrutiny in cells with abolished expression of ataxin-7 and in SCA7-null mice.

Protein recruitment into NI Nuclear aggregates of polyglutamine disease proteins recruit a variety of additional cellular proteins. Sequestration of these proteins likely interferes with their functions, thereby contributing to toxicity. While some cellular factors will recognize structural features common to aggregates of all polyglutamine disease proteins, other factors will be recruited into aggregates by means of their interaction with the nonpolyglutamine portion of the aggregated disease proteins. Hence, aggregates of distinct disease proteins will sequester overlapping, but nonidentical sets of cellular proteins, perhaps explaining why select neuronal types are preferentially affected in each disorder. ${ }^{71}$

As expected, SCA7 NI comprise many of the proteins found in aggregates of other polyglutamine disease proteins, including the molecular chaperones HDJ-2/ Hsp40 and Hsp70, ubiquitin, and the proteasome, and the transcriptional coactivator CBP. ${ }^{62,63,65,66,69,72}$ To pinpoint novel components of SCA7 NI, Yvert et al ${ }^{69}$ inspected colocalization into NI of 18 nuclear proteins involved in transcriptional regulation in the B7E SCA7 transgenic mice. Of these, only the basal factors TFIIE $\alpha$ and TFIIF $\beta$, the transcriptional coactivator CBP, and the TAFII30 subunit of the TFIID initiation complex were frequently noticed in the inclusions. Importantly, however, despite their recruitment into NI, the levels of these proteins in the nucleoplasm did not appear reduced, thus questioning whether their function would in fact be compromised. Clearly, further studies need to address the extent of transcriptional deregulation in transgenic models of SCA7. Notably, the study of Yvert et al did not detect any differences in composition of SCA7 NI between affected and spared neurons of the B7E transgenic mice. Nevertheless, there might exist other, as yet unidentified proteins, which will either be sequestered into SCA7 NI exclusively in affected neurons, or alternatively, recruitment of which will only be harmful to SCA7-vulnerable neurons.

In conclusion, we outlined several mechanisms as to how the nonpolyglutamine sequences of ataxin-7 might modulate SCA7 pathogenesis. While none of the above mechanisms can on its own account for the restricted pattern of neuronal dysfunction and degeneration in SCA7, this will likely result from interplay of several of 
these factors. To gain a deeper insight into the processes involved in SCA7 pathogenesis, researchers resorted to the study of transgenic models of SCA7. Analysis of transgenic animals offers a unique chance to pin down the molecular events initiating neuronal dysfunction, as well as to unravel the precise sequence of functional and morphological alterations accompanying SCA7 progression. In the following, we review the most relevant findings of these studies.

\section{SCA7 animal models}

The retina To reproduce the characteristic SCA7 retinopathy, Yvert et $a l^{63}$ generated transgenic mice (R7E) selectively overexpressing mutant ataxin-7 (90Q) in rod photoreceptors. The animals suffered progressive impairment of photoreceptor function and showed degenerative changes in the retina, including loss of the outer and inner segments and an irregular 'wavy' aspect of the outer nuclear layer (ONL). Importantly, visual function was markedly reduced before overt histological abnormalities were noted in the retina, arguing that photoreceptor dysfunction rather than degeneration underlies the visual failure in SCA7. Appearance of NI in photoreceptors of these mice coincided with the onset of functional deficits in these cells, suggesting that nuclear accumulation and aggregation of mutant ataxin-7 instigates this process.

To approximate more closely the situation in the retina of SCA7 patients, La Spada et $a l^{73}$ generated transgenic mice (PrP-SCA7-c92Q), expressing mutant ataxin-7 in both cone and rod photoreceptors, as well as in the inner layers of the retina. The mice developed a cone-rod dystrophy phenotype similar to SCA7, as evidenced by early decline in function of cones and only later involvement of rod photoreceptors. Nevertheless, this eventually progressed toward complete loss of both cone and rod function and blindness. Degenerative changes in the retina included periodic thinning of the ONL, apoptosis of cone photoreceptors, and gliosis. However, marked malfunction of photoreceptors was noted in animals with histologically normal retina and only minimal pathological changes were observed in the retina of completely blind animals. This further corroborates the notion that visual failure in SCA7 results from dysfunction rather than demise of photoreceptors. Mutant ataxin-7 formed prominent NI in all three nuclear layers of the retina in these mice. However, the study did not mention any functional or histological abnormalities in bipolar and ganglion neurons of these animals.

La Spada et $a l^{73}$ hypothesized that impaired function of the cone-rod homeobox protein (CRX) - a photoreceptorspecific transcription factor - might underlie the characteristic retinopathy of SCA7. Mutations in CRX cause cone-rod dystrophy in humans and CRX-null mice develop progressive retinal degeneration. Importantly, CRX contains a glutamine-rich region, which could potentially bind the expanded polyglutamine tract in ataxin-7. The authors investigated whether such interaction actually occurred, and found that both wild-type and mutant ataxin-7 interacted with CRX. Moreover, the mutant protein greatly interfered with CRX-mediated transcription. This effect was also reproduced in vivo expression levels of several CRX-controlled genes were reduced in the retina of the PrP-SCA7-C92Q mice before the onset of photoreceptor dysfunction. Notably, this reduction was significantly greater for cone-specific than for rodspecific genes, in agreement with CRX being more essential for transcription of the former. Thus, CRX dysfunction would preferentially affect cone photoreceptors, perhaps explaining the distinctive cone-rod dystrophy phenotype of SCA7. However, this hypothesis was not corroborated in a recent SCA7 knock-in model (see below).

To study the consequences of mutant ataxin-7 expression at endogenous levels, Yoo et $a l^{74}$ generated SCA7 knock-in mice carrying 266 glutamines. The animals displayed progressive deterioration of photoreceptor responses, with the cones being affected earlier and more severely than the rods. These functional deficits advanced in parallel with shortening of the photosensitive outer segments of the photoreceptors. On the contrary, the organization of the ONL remained largely intact, and the extent of photoreceptor apoptosis was rather limited. Once again, this emphasizes the key role of photoreceptor dysfunction - as opposed to photoreceptor demise - in the SCA7 visual impairment. Mutant ataxin-7 progressively accumulated in photoreceptor nuclei, but formed visible structures only after the onset of functional aberrations. Since no soluble ataxin-7 was detected in these mice even at an early age, this indicates that submicroscopic aggregates of ataxin-7 might initiate the pathogenesis.

Yoo et $a l^{74}$ next inspected whether transcriptional deregulation might be responsible for photoreceptor malfunction in the SCA7 knock-in mice. Indeed, many critical photoreceptor genes turned out to be dramatically downregulated in presymptomatic animals. However, the observed pattern of gene expression changes was not consistent with CRX deficiency being the sole initiator of these alterations - the SCA7 knockin retinas showed downregulation of several genes, which are normally unaffected or even upregulated in response to CRX deletion. Moreover, the authors could not demonstrate any reduction in CRX levels, or in its DNA-binding ability in the SCA7 knockin animals. Therefore, the visual phenotype of SCA7 is likely brought about by a general downregulation of many critical photoreceptor genes, not only those regulated by CRX.

Recently, Helmlinger et $a l^{75}$ addressed the role of ataxin-7 protein context in specifying the retinal involvement in SCA7. These authors reported that transgenic mice expressing truncated, polyglutamine-expanded huntingtin 
(the protein mutated in HD) also developed retinal dysfunction. Intriguingly, the function of cones was compromised prior to that of rods, indicating that expanded polyglutamine can induce cone-rod dystrophy outside of the ataxin-7 context. Yet, it remains to be seen whether the molecular pathways underlying the visual defects in these mice are identical to those in the SCA7 animals.

The cerebellum To replicate the cerebellar phenotype of SCA7, Yvert et $a l^{63}$ targeted expression of mutant ataxin-7 with 90 glutamines selectively into Purkinje cells of transgenic mice. However, the animals did not develop overt ataxia and displayed only mild morphological alterations of Purkinje cells. Although these findings are surprising, given the extreme vulnerability of Purkinje neurons in SCA7, they are most likely attributable to insufficient expression of the mutant protein in this model. Indeed, when ataxin-7 carrying an identical number of glutamines was expressed at higher levels using the PDGF promoter, the resulting mice (B7E) became visibly ataxic. ${ }^{69}$ Intriguingly, ataxia was the most prominent phenotypic feature, despite the widespread expression and aggregation of the mutant protein in these animals.

The SCA7 knockin mice harboring 266 glutamines in endogenous ataxin-7, ${ }^{74}$ developed a striking motor phenotype, starting as a subtle incoordination on rotarod testing, but progressing toward visible ataxia, and eventually to hypo activity in terminal stages. Importantly, the mice did not suffer any Purkinje cell loss, suggesting that ataxia in SCA7 might result primarily from neuronal dysfunction, while neuronal demise would follow later. Visible nuclear inclusions in Purkinje cells formed only after the onset of ataxia, further emphasizing the role of submicroscopic aggregates in initiating the SCA7 pathogenesis. Intriguingly, the neuronal types most severely affected in these mice (and in SCA7) - the retinal photoreceptors and the cerebellar neurons - were among the first to develop discernible NI, indicating that they might experience accelerated accumulation of insoluble ataxin-7.

An interesting twist was provided by analyzing the motor phenotype in PrP-SCA7-c92Q mice. ${ }^{76}$ In these mice, mutant ataxin-7 is expressed in most CNS neurons with the exception of cerebellar Purkinje cells. Nonetheless, the mice do develop ataxia and display shrunken, abnormally shaped Purkinje cells with reduced arborization. This argues that expression of mutant ataxin-7 in neurons connecting to Purkinje cells can provoke noncell autonomous dysfunction and morphological abnormalities in the latter. While neuronal populations suffering the primary insult are unknown, these could include cerebellar granule neurons, non-Purkinje neurons of the molecular layer, or neurons of inferior olivary and pontine nuclei. Notably, nuclear accumulation and aggregation of ataxin-7 in these neurons coincides with the onset of ataxia and Purkinje cell alterations in PrP-SCA7-c92Q mice.

In summary, the animal models offer the following picture of the early events in SCA7 pathogenesis. Expansion of the polyglutamine tract in ataxin-7 markedly reduces the turnover of the mutant protein, thus increasing its levels in neurons. Next, mutant ataxin-7 undergoes proteolytic cleavage and its $\mathrm{N}$-terminal polyglutaminecontaining fragments gradually accumulate and eventually aggregate in the nucleus. This nuclear accumulation and aggregation of ataxin-7 fragments coincides with the onset of functional deficits in affected neurons, indicating that mutant ataxin-7 compromises essential nuclear functions. Importantly, neuronal loss is noted only after an extended period of neuronal dysfunction, arguing that the SCA7 phenotype results primarily from functional decline rather than demise of neurons. Last but not least, transneuronal effects might significantly widen the scale of neuronal deterioration in SCA7.

Not surprisingly, the above scenario raises more questions than it provides answers. To begin with, what renders mutant ataxin-7 more resistant to degradation than its wild-type counterpart? Are elevated levels of full-length mutant ataxin-7 injurious to neurons, and if so, what is the mechanism of such toxicity? Does the partial proteolysis of mutant ataxin-7 result from its disproportionate accumulation, or is proteolytic cleavage integral to the metabolism of the wild-type protein as well? In either case, which protease(s) catalyze the cleavage of mutant ataxin-7 and where precisely in ataxin-7 do they cut? Above all, the role of proteases known to cleave other polyglutamine disease proteins, such as caspases, ${ }^{77}$ calpains, ${ }^{78}$ and aspartic endopeptidases, ${ }^{79}$ should be thoroughly investigated. Furthermore, why does truncated mutant ataxin-7 gradually accumulate within the nuclei instead of being cleared by the proteasome? Since intranuclear accumulation of expanded-polyglutamine proteins is also noted in animal models of other polyglutamine disorders, ${ }^{80-82}$ this question is central to understanding polyglutamine pathogenesis. Undeniably, the most challenging task will be to pinpoint the nuclear processes disrupted by mutant ataxin-7, and especially those selectively compromised in SCA7-vulnerable neurons. Among these, ataxin-7's interference with CRX-mediated transcription might underlie the cone-rod dystrophy in SCA7, although this finding remains controversial. Finally, normal cellular function of ataxin-7 needs to be deciphered, in order to assess whether loss of function adds to SCA7 pathogenesis. Certainly, unraveling these issues will require substantial scientific effort over many years to come. Nevertheless, the existence of suitable animal models of SCA7 will greatly facilitate testing of novel hypotheses concerning the mechanisms of initiation and progression of the SCA7 pathogenesis. 
Acknowledgements

The Fund for Scientific Research Flanders, Belgium, has funded the research on SCA7 at our department of Molecular Genetics. We greatly appreciate the contributions to the research project of $N$ Van Regemorter and A Löfgren.

\section{References}

1 Froment J, Bonnet P, Colrat A: Heredo-degenerations retinienne et spino-cerebelleuse: variantes ophtalmoscopiques et neurologiques presentees par trois generations successives. J Med Lyon 1937; $153-163$.

2 Harding AE: The clinical features and classification of the late onset autosomal dominant cerebellar ataxia: A study of 11 families, including descendants of the Drew family of Walworth'. Brain 1982; 105: 1-28.

3 Konigsmark BW, Weiner LP: The olivopontocerebellar atrophies: a review. Medicine 1970; 49: 227-241.

4 Enevoldson TP, Sanders MD, Harding AE: Autosomal dominant cerebellar ataxia with pigmentary macular dystrophy. A clinical and genetic study of eight families. Brain 1994; 117: 445-460.

5 Martin JJ, Van Regemorter N, Krols L, Brucher JM, de Barsy T, Szliwowski $\mathrm{H}$ : On an autosomal dominant form of retinalcerebellar degeneration: an autopsy study of five patients in one family. Acta Neuropathol (Berl) 1994; 88: 277-286.

6 Benomar A, Le Guern E, Durr A, Ouhabi H, Stevanin G, Yahyaoui M: Autosomal-dominant cerebellar ataxia with retinal degeneration (ADCA type II) is genetically different from ADCA type I. Ann Neurol 1994; 35: 439-444.

7 Gouw LG, Digre KB, Harris CP, Haines JH, Ptacek LJ: Autosomal dominant cerebellar ataxia with retinal degeneration: clinical, neuropathologic, and genetic analysis of a large kindred. Neurology 1994; 44: 1441-1447.

8 Holmberg M, Johansson J, Forsgren L, Heijbel J, Sandgren O, Holmgren G: Localization of autosomal dominant cerebellar ataxia associated with retinal degeneration and anticipation to chromosome 3p12-p21.1. Hum Mol Genet 1995; 4: 1441-1445.

9 Jobsis GJ, Weber JW, Barth PG, Keizers H, Baas F, van Schooneveld MJ: Autosomal dominant cerebellar ataxia with retinal degeneration (ADCA II): clinical and neuropathological findings in two pedigrees and genetic linkage to 3p12-p21.1. I Neurol Neurosurg Psychiatry 1997; 62: 367-371.

10 David G, Durr A, Stevanin G, Cancel G, Abbas N, Benomar A: Molecular and clinical correlations in autosomal dominant cerebellar ataxia with progressive macular dystrophy (SCA7). Hum Mol Genet 1998; 7: 165-170.

11 Gouw LG, Castaneda MA, McKenna CK, Digre KB, Pulst SM, Perlman S: Analysis of the dynamic mutation in the SCA7 gene shows marked parental effects on CAG repeat transmission. Hum Mol Genet 1998; 7: 525-532.

12 Johansson J, Forsgren L, Sandgren O, Brice A, Holmgren G, Holmberg M: Expanded CAG repeats in Swedish spinocerebellar ataxia type 7 (SCA7) patients: effect of CAG repeat length on the clinical manifestation. Hum Mol Genet 1998; 7: 171-176.

13 Giunti P, Stevanin G, Worth PF, David G, Brice A, Wood NW: Molecular and clinical study of 18 families with ADCA type II: evidence for genetic heterogeneity and de novo mutation. Am J Hum Genet 1999; 64: 1594-1603.

14 Martin J, Van Regemorter N, Del Favero J, Lofgren A, van Broeckhoven C: Spinocerebellar ataxia type 7 (SCA7) correlations between phenotype and genotype in one large Belgian family. J Neurol Sci 1999; 168: 37-46.

15 Benomar A, Krols L, Stevanin G, Cancel G, LeGuern E, David G: The gene for autosomal dominant cerebellar ataxia with pigmentary macular dystrophy maps to chromosome 3p12-p21.1. Nat Genet 1995; 10: 84-88.

16 van de Warrenburg BP, Frenken CW, Ausems MG, Kleefstra T, Sinke RJ, Knoers NV: Striking anticipation in spinocerebellar ataxia type 7: the infantile phenotype. J Neurol 2001; 248: 911914.

17 Benton CS, de Silva R, Rutledge SL, Bohlega S, Ashizawa T, Zoghbi HY: Molecular and clinical studies in SCA-7 define a broad clinical spectrum and the infantile phenotype. Neurology 1998; 51: $1081-1086$

18 Gouw LG, Kaplan CD, Haines JH, Digre KB, Rutledge SL, Matilla A: Retinal degeneration characterizes a spinocerebellar ataxia mapping to chromosome 3p. Nat Genet 1995; 10: 89-93.

19 David G, Giunti P, Abbas N, Coullin P, Stevanin G, Horta W: The gene for autosomal dominant cerebellar ataxia type II is located in a 5-cM region in 3p12-p13: genetic and physical mapping of the SCA7 locus. Am J Hum Genet 1996; 59: 1328-1336.

20 Krols L, Martin JJ, David G, Van Regemorter N, Benomar A Lofgren A: Refinement of the locus for autosomal dominant cerebellar ataxia type II to chromosome 3p21.1-14.1. Hum Genet 1997; 99: 225-232.

21 La Spada AR, Paulson HL, Fischbeck KH: Trinucleotide repeat expansion in neurological disease. Ann Neurol 1994; 36: 814-822.

22 Lindblad K, Savontaus ML, Stevanin G, Holmberg M, Digre K, Zander C: An expanded CAG repeat sequence in spinocerebellar ataxia type 7. Genome Res 1996; 6: 965-971.

23 Trottier Y, Lutz Y, Stevanin G, Imbert G, Devys D, Cancel G: Polyglutamine expansion as a pathological epitope in Huntington's disease and four dominant cerebellar ataxias. Nature 1995; 378: 403-406.

24 Stevanin G, Trottier Y, Cancel G, Durr A, David G, Didierjean O: Screening for proteins with polyglutamine expansions in autosomal dominant cerebellar ataxias. Hum Mol Genet 1996; 5: $1887-1892$.

25 David G, Abbas N, Stevanin G, Durr A, Yvert G, Cancel G: Cloning of the SCA7 gene reveals a highly unstable CAG repeat expansion. Nat Genet 1997; 17: 65-70.

26 Del Favero J, Krols L, Michalik A, Theuns J, Lofgren A, Goossens D: Molecular genetic analysis of autosomal dominant cerebellar ataxia with retinal degeneration (ADCA type II) caused by CAG triplet repeat expansion. Hum Mol Genet 1998; 7: 177-186.

27 Koob MD, Benzow KA, Bird TD, Day JW, Moseley ML, Ranum LP: Rapid cloning of expanded trinucleotide repeat sequences from genomic DNA. Nat Genet 1998; 18: 72-75.

28 Michalik A, Del Favero J, Mauger C, Lofgren A, van Broeckhoven C: Genomic organisation of the spinocerebellar ataxia type 7 (SCA7) gene responsible for autosomal dominant cerebellar ataxia with retinal degeneration. Hum Genet 1999; 105: 410-417.

29 Takano H, Cancel G, Ikeuchi T, Lorenzetti D, Mawad R, Stevanin G: Close associations between prevalences of dominantly inherited spinocerebellar ataxias with CAG-repeat expansions and frequencies of large normal CAG alleles in Japanese and Caucasian populations. Am J Hum Genet 1998; 63: $1060-1066$

30 Moseley ML, Benzow KA, Schut LJ, Bird TD, Gomez CM, Barkhaus PE: Incidence of dominant spinocerebellar and Friedreich triplet repeats among 361 ataxia families. Neurology 1998; 51: $1666-1671$.

31 Pujana MA, Corral J, Gratacos M, Combarros O, Beroiano J, Genis D: Spinocerebellar ataxias in Spanish patients: genetic analysis of familial and sporadic cases. The Ataxia Study Group. Hum Genet 1999; 104: 516-522.

32 Filla A, Mariotti C, Caruso G, Coppola G, Cocozza S, Castaldo I: Relative frequencies of CAG expansions in spinocerebellar ataxia and dentatorubropallidoluysian atrophy in 116 Italian families. Eur Neurol 2000; 44: 31-36.

33 Storey E, du Sart D, Shaw JH, Lorentzos P, Kelly L, McKinley Gardner RJ: Frequency of spinocerebellar ataxia types 1, 2, 3, 6, and 7 in Australian patients with spinocerebellar ataxia. Am J Med Genet 2000; 95: 351-357.

34 Soong BW, Lu YC, Choo KB, Lee HY: Frequency analysis of autosomal dominant cerebellar ataxias in Taiwanese patients and clinical and molecular characterization of spinocerebellar ataxia type 6. Arch Neurol 2001; 58: 1105-1109. 
35 Jardim LB, Silveira I, Pereira ML, Ferro A, Alonso I, do Ceu MM: A survey of spinocerebellar ataxia in South Brazil - 66 new cases with Machado-Joseph disease, SCA7, SCA8, or unidentified disease-causing mutations. J Neurol 2001; 248: 870-876.

36 Kim JY, Park SS, Joo SI, Kim JM, Jeon BS: Molecular analysis of Spinocerebellar ataxias in Koreans: frequencies and reference ranges of SCA1, SCA2, SCA3, SCA6, and SCA7. Mol Cells 2001; 12: $336-341$.

37 van de Warrenburg BP, Sinke RJ, Verschuuren-Bemelmans CC, Scheffer H, Brunt ER, Ippel PF: Spinocerebellar ataxias in the Netherlands: prevalence and age at onset variance analysis. Neurology 2002; 58: 702-708.

38 Silveira I, Miranda C, Guimaraes L, Moreira MC, Alonso I, Mendonca P: Trinucleotide repeats in 202 families with ataxia: a small expanded (CAG) $n$ allele at the SCA17 locus. Arch Neurol 2002; 59: 623-629.

39 Jonasson J, Juvonen V, Sistonen P, Ignatius J, Johansson D, Bjorck EJ: Evidence for a common Spinocerebellar ataxia type 7 (SCA7) founder mutation in Scandinavia. Eur J Hum Genet 2000; 8: 918-922.

40 Zoghbi HY, Orr HT: Glutamine repeats and neurodegeneration. Annu Rev Neurosci 2000; 23: 217-247.

41 Frontali M: Spinocerebellar ataxia type 6: channelopathy or glutamine repeat disorder? Brain Res Bull 2001; 56: 227-231.

42 Stevanin G, Giunti P, Belal GD, Durr A, Ruberg M, Wood N: De novo expansion of intermediate alleles in spinocerebellar ataxia 7. Hum Mol Genet 1998; 7: 1809-1813.

43 Nardacchione A, Orsi L, Brusco A, Franco A, Grosso E, Dragone E: Definition of the smallest pathological CAG expansion in SCA7. Clin Genet 1999; 56: 232-234.

44 Monckton DG, Cayuela ML, Gould FK, Brock GJ, Silva R, Ashizawa T: Very large (CAG)(n) DNA repeat expansions in the sperm of two spinocerebellar ataxia type 7 males. Hum Mol Genet 1999; 8: 2473-2478.

45 Brock GJ, Anderson NH, Monckton DG: Cis-acting modifiers of expanded CAG/CTG triplet repeat expandability: associations with flanking GC content and proximity to $\mathrm{CpG}$ islands. Hum Mol Genet 1999; 8: 1061-1067.

46 Libby RT, Monckton DG, Fu YH, Martinez RA, McAbney JP, Lau R: Genomic context drives SCA7 CAG repeat instability, while expressed SCA7 cDNAs are intergenerationally and somatically stable in transgenic mice. Hum Mol Genet 2003; 12: 41-50.

47 Lebre AS, Jamot L, Takahashi J, Spassky N, Leprince C, Ravise N: Ataxin-7 interacts with a Cbl-associated protein that it recruits into neuronal intranuclear inclusions. Hum Mol Genet 2001; 10: $1201-1213$.

48 Kaytor MD, Duvick LA, Skinner PJ, Koob MD, Ranum LP, Orr HT: Nuclear localization of the spinocerebellar ataxia type 7 protein, ataxin-7. Hum Mol Genet 1999; 8: 1657-1664.

49 Mushegian AR, Vishnivetskiy SA, Gurevich VV: Conserved phosphoprotein interaction motif is functionally interchangeable between ataxin-7 and arrestins. Biochemistry 2000; 39: 6809-6813.

50 Strom AL, Jonasson J, Hart P, Brannstrom T, Forsgren L, Holmberg M: Cloning and expression analysis of the murine homolog of the spinocerebellar ataxia type 7 (SCA7) gene. Gene 2002; 285: 91-99.

51 Paulson HL: Toward an understanding of polyglutamine neurodegeneration. Brain Pathol 2000; 10: 293-299.

52 Gusella JF, MacDonald ME: Molecular genetics: unmasking polyglutamine triggers in neurodegenerative disease. Nat Rev Neurosci 2000; 1: 109-115.

53 Tobin AJ, Signer ER: Huntington's disease: the challenge for cell biologists. Trends Cell Biol 2000; 10: 531-536.

54 Ross CA: When more is less: pathogenesis of glutamine repeat neurodegenerative diseases. Neuron 1995; 15: 493-496.

55 Lindenberg KS, Yvert G, Muller K, Landwehrmeyer GB: Expression analysis of ataxin-7 mRNA and protein in human brain: evidence for a widespread distribution and focal protein accumulation. Brain Pathol 2000; 10: 385-394.
56 Cancel G, Duyckaerts C, Holmberg M, Zander C, Yvert G, Lebre AS: Distribution of ataxin-7 in normal human brain and retina. Brain 2000; 123: 2519-2530.

57 Einum DD, Townsend JJ, Ptacek LJ, Fu YH: Ataxin-7 expression analysis in controls and spinocerebellar ataxia type 7 patients. Neurogenetics 2001; 3: 83-90.

58 Jonasson J, Strom AL, Hart P, Brannstrom T, Forsgren L, Holmberg M: Expression of ataxin-7 in CNS and non-CNS tissue of normal and SCA7 individuals. Acta Neuropathol (Berl) 2002; 104: 29-37.

59 Klement IA, Skinner PJ, Kaytor MD, Yi H, Hersch SM, Clark HB Ataxin-1 nuclear localization and aggregation: role in polyglutamine-induced disease in SCA1 transgenic mice. Cell 1998; 95: 41-53.

60 Saudou F, Finkbeiner S, Devys D, Greenberg ME: Huntingtin acts in the nucleus to induce apoptosis but death does not correlate with the formation of intranuclear inclusions. Cell 1998; 95: $55-66$.

61 Peters MF, Nucifora FC, Kushi J, Seaman HC, Cooper JK, Herring WJ: Nuclear targeting of mutant Huntingtin increases toxicity. Mol Cell Neurosci 1999; 14: 121-128.

62 Zander C, Takahashi J, El Hachimi KH, Fujigasaki H, Albanese V, Lebre AS: Similarities between spinocerebellar ataxia type 7 (SCA7) cell models and human brain: proteins recruited in inclusions and activation of caspase-3. Hum Mol Genet 2001; 10: 2569-2579.

63 Yvert G, Lindenberg KS, Picaud S, Landwehrmeyer GB, Sahel JA, Mandel JL: Expanded polyglutamines induce neurodegeneration and trans-neuronal alterations in cerebellum and retina of SCA7 transgenic mice. Hum Mol Genet 2000; 9: 2491-2506.

64 Einum DD, Clark AM, Townsend JJ, Ptacek LJ, Fu YH: A novel central nervous system-enriched spinocerebellar ataxia type 7 gene product. Arch Neurol 2003; 60: 97-103.

65 Holmberg M, Duyckaerts C, Durr A, Cancel G, Gourfinkel-An I, Damier P: Spinocerebellar ataxia type 7 (SCA7): a neurodegenerative disorder with neuronal intranuclear inclusions. Hum Mol Genet 1998; 7: 913-918.

66 Mauger C, Del Favero J, Ceuterick C, Lubke U, van Broeckhoven $\mathrm{C}$, Martin J: Identification and localization of ataxin-7 in brain and retina of a patient with cerebellar ataxia type II using antipeptide antibody. Brain Res Mol Brain Res 1999; 74: 35-43.

67 Chen S, Berthelier V, Yang W, Wetzel R: Polyglutamine aggregation behavior in vitro supports a recruitment mechanism of cytotoxicity. J Mol Biol 2001; 311: 173-182.

68 Cummings CJ, Reinstein E, Sun Y, Antalffy B, Jiang Y, Clechanover A: Mutation of the E6-AP ubiquitin ligase reduces nuclear inclusion frequency while accelerating polyglutamineinduced pathology in SCA1 mice. Neuron 1999; 24: 879-892.

69 Yvert G, Lindenberg KS, Devys D, Helmlinger D, Landwehrmeyer GB, Mandel JL: SCA7 mouse models show selective stabilization of mutant ataxin-7 and similar cellular responses in different neuronal cell types. Hum Mol Genet 2001; 10: 1679-1692.

70 Matilla A, Gorbea C, Einum DD, Townsend J, Michalik A, van Broeckhoven C: Association of ataxin-7 with the proteasome subunit S4 of the 19S regulatory complex. Hum Mol Genet 2001; 10: $2821-2831$

71 Chai Y, Wu L, Griffin JD, Paulson HL: The role of protein composition in specifying nuclear inclusion formation in polyglutamine disease. J Biol Chem 2001; 276: 44889-44897.

72 Takahashi J, Fujigasaki H, Zander C, El Hachimi KH, Stevanin G, Durr A: Two populations of neuronal intranuclear inclusions in SCA7 differ in size and promyelocytic leukaemia protein content. Brain 2002; 125: 1534-1543.

73 La Spada AR, Fu YH, Sopher BL, Libby RT, Wang X, Li LY: Polyglutamine-expanded ataxin-7 antagonizes CRX function and induces cone-rod dystrophy in a mouse model of SCA7. Neuron 2001; 31: 913-927.

74 Yoo SY, Pennesi ME, Weeber EJ, Xu B, Atkinson R, Chen S: SCA7 knockin mice model human SCA7 and reveal gradual accumulation of mutant ataxin-7 in neurons and abnormalities in short-term plasticity. Neuron 2003; 37: 383-401. 
75 Helmlinger D, Yvert G, Picaud S, Merienne K, Sahel J, Mandel JL: Progressive retinal degeneration and dysfunction in R6 Huntington' disease mice. Hum Mol Genet 2002; 11: 3351-3359.

76 Garden GA, Libby RT, Fu YH, Kinoshita Y, Huang J, Possin DE: Polyglutamine-expanded ataxin-7 promotes non-cellautonomous Purkinje cell degeneration and displays proteolytic cleavage in ataxic transgenic mice. J Neurosci 2002; 22: 4897-4905.

77 Wellington CL, Ellerby LM, Hackam AS, Margolis RL, Trifiro MA, Singaraja R: Caspase cleavage of gene products associated with triplet expansion disorders generates truncated fragments containing the polyglutamine tract. J Biol Chem 1998; 273: 9158-9167.

78 Kim YJ, Yi Y, Sapp E, Wang Y, Cuiffo B, Kegel KB: Caspase 3cleaved N-terminal fragments of wild-type and mutant huntingtin are present in normal and Huntington's disease brains, associate with membranes, and undergo calpain- dependent proteolysis. Proc Natl Acad Sci USA 2001; 98 : 12784-12789.

79 Lunkes A, Lindenberg KS, Ben Haiem L, Weber C, Devys D, Landwehrmeyer GB: Proteases acting on mutant huntingtin generate cleaved products that differentially build up cytoplasmic and nuclear inclusions. Mol Cell 2002; 10: 259-269.

80 Davies SW, Turmaine M, Cozens BA, DiFiglia M, Sharp AH, Ross CA: Formation of neuronal intranuclear inclusions underlies the neurological dysfunction in mice transgenic for the HD mutation. Cell 1997; 90: 537-548.

81 Li H, Li SH, Johnston H, Shelbourne PF, Li XJ: Amino-terminal fragments of mutant huntingtin show selective accumulation in striatal neurons and synaptic toxicity. Nat Genet 2000; 25: $385-389$.

82 Schilling G, Wood JD, Duan K, Slunt HH, Gonzales V, Yamada M: Nuclear accumulation of truncated atrophin-1 fragments in a transgenic mouse model of DRPLA. Neuron 1999; 24: 275-286. 